\title{
Trends in Intravenous Magnesium Use and Outcomes for Status Asthmaticus in Children's Hospitals from 2010 to 2017
}

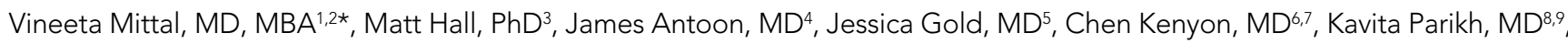 \\ Rustin Morse, MD ${ }^{10}$, Ricardo Quinonez, MD ${ }^{11,12}$, Ronald J Teufel II, MD ${ }^{13}$, Samir S Shah, MD ${ }^{14}$
}

${ }^{1}$ Division of Pediatric Hospital Medicine, Department of Pediatrics, UT Southwestern Medical Center, Dallas, Texas; ${ }^{2}$ Department of Pediatrics, Children's Health System, Dallas, Texas; ${ }^{3}$ Informatics, Children's Hospital Association, Lenexa, Kansas; ${ }^{4}$ Division of Pediatric Hospital Medicine, Department of Pediatrics, Vanderbilt University School of Medicine; ${ }^{5}$ Pediatrics, Stanford University School of Medicine/Lucile Packard Children's Hospital, San Mateo, California; ${ }^{6}$ Department of Pediatrics, Children's Hospital of Pennsylvania, Philadelphia, Pennsylvania; ${ }^{7}$ Department of Pediatrics, Perelman School of Medicine, University of Pennsylvania, Philadelphia, Pennsylvania; ${ }^{8}$ Hospitalist Division, Children's National Medical Center, Washington, District of Columbia; ${ }^{9}$ Hospitalist Division, George Washington School of Medicine, Washington, District of Columbia; ${ }^{10}$ Department of Quality and Safety, Children's Health System of Texas, Dallas, Texas; ${ }^{11}$ Division of Pediatric Hospital Medicine, Texas Children's Hospital, Houston, Texas; ${ }^{12}$ Division of Pediatric Hospital Medicine, Baylor College of Medicine, Houston, Texas; ${ }^{13}$ Departemnt of Pediatrics, Medical University of South Carolina, Charleston, South Carolina; ${ }^{14}$ Division of Pediatric Hospital Medicine, Department of Pediatrics, Cincinnati Children's Hospital Medical Center, Cincinnati, Ohio.

Intravenous (IV) magnesium is used as an adjunct therapy in management of status asthmaticus with a goal of reducing intubation rate. A recent review suggests that IV magnesium use in status asthmaticus reduces admission rates. This is contrary to the observation of practicing emergency room physicians. The goal of this study was to assess trends in IV magnesium use for status asthmaticus in US children's hospitals over 8 years through a retrospective analysis of children younger than 18 years using the Pediatric Health Information System database.
Outcomes were IV magnesium use, inpatient and intensive care unit admission rate, geometric mean length of stay, and 7-day all-cause readmission rate. IV magnesium use for asthma hospitalization more than doubled over 8 years $(17 \%$ vs. $36 \% ; P<.001)$. Yearly trends were not significantly associated with hospital or intensive care unit admission rate or 7-day all-cause readmissions, although length of stay was reduced $(P<.001)$. Journal of Hospital Medicine 2020;15:403-406. C2020 Society of Hospital Medicine

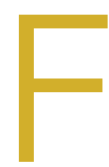

or severe asthma exacerbations unresponsive to initial treatment, expert consensus guidelines from 2007 recommend consideration for adjunct treatments (magnesium or heliox) to decrease the likelihood of intubation. ${ }^{1}$ Over the last decade, data have emerged suggesting that intravenous (IV) magnesium may be more effective for reduction of hospital admission rates. ${ }^{2}$ Pooled meta-analyses have demonstrated improved pulmonary function and reduction of hospital admission by as much as $68 \%$ in children when IV magnesium is administered in the emergency department (ED), although the evidence is extremely limited because of a small number of studies (three) and small sample size (115 children). ${ }^{2-5}$

Though these data suggest that use of IV magnesium may reduce admission rates, a study of pediatric emergency medicine (PEM) physicians in US and Canada reported reluctance regarding use for this purpose. While PEM physicians reported awareness of the literature on admission prevention, they estimated

*Corresponding Author: Vineeta Mittal, MD, MBA; Email: Vineeta.Mittal@ UTSouthwestern.edu; Telephone: 214-456-5527; Twitter: @Vmittal.

Published online first June 17, 2020.

Find additional supporting information in the online version of this article.

Received: July 7, 2019; Revised: March 2, 2020; Accepted: March 4, 2020

(c) 2020 Society of Hospital Medicine DOI 10.12788/jhm.3405 that fewer than $5 \%$ of their patients receiving IV magnesium were discharged home. ${ }^{6}$ Their practice was generally limited to using IV magnesium in children with impending respiratory failure for the purpose of reducing intensive care unit (ICU) admission and not hospitalization. ${ }^{6}$ PEM physicians' reluctance to use IV magnesium was related to the lack of strong available evidence supporting the impact of IV magnesium on outcomes, such as admission, and gaps in the literature about its dosing and safety profile.

The goal of this study was to assess the prevailing trends in IV magnesium use across US children's hospitals and to assess the relationship of IV magnesium use to admission rate, length of stay (LOS), readmission rate, and ICU admission rate. We hypothesized that IV magnesium use might have increased following publication of studies demonstrating an association between IV magnesium use and fewer admissions.

\section{METHODS}

\section{Study Design, Setting, and Participants}

This is a retrospective cohort study of asthma (All Patient Refined Diagnosis Related Group 141) hospitalizations for patients less than 18 years old presenting to 35 tertiary care children's hospitals from January 1, 2010, to December 31, 2017, included in the Pediatric Health Information System (PHIS; Children's Hospital Association, Lenexa, Kansas) database. 
TABLE. Social Demographics of Participants

\begin{tabular}{|c|c|c|c|c|c|c|c|}
\hline & & \multicolumn{2}{|c|}{ Combined } & \multicolumn{2}{|c|}{ Admitted } & \multicolumn{2}{|c|}{ Not Admitted } \\
\hline & & No Mag & Mag & No Mag & Mag & No Mag & Mag \\
\hline \multicolumn{2}{|l|}{$\mathrm{N}(\%)$} & $812,630(92.5)$ & $65,558(7.5)$ & $168,889(74.1)$ & $59,069(25.9)$ & $643,741(99.0)$ & $6,489(1.0)$ \\
\hline \multicolumn{2}{|c|}{ Age: Median [Interquartile Range] } & $5[3,9]$ & $6[3,10]$ & $4[2,8]$ & $6[3,10]$ & $5[3,9]$ & $6[4,10]$ \\
\hline \multirow[t]{3}{*}{ Age group } & $<5$ & $36,7726(45.3)$ & $23,399(35.7)$ & $87,089(51.6)$ & $21,268(36)$ & $280,637(43.6)$ & $2,131(32.8)$ \\
\hline & $5-11$ & $34,5624(42.5)$ & $32,063(48.9)$ & $65,628(38.9)$ & $28,813(48.8)$ & $279,996(43.5)$ & $3,250(50.1)$ \\
\hline & $12-17$ & $99,280(12.2)$ & $10,096(15.4)$ & $16,172(9.6)$ & $8,988(15.2)$ & $83,108(12.9)$ & $1,108(17.1)$ \\
\hline \multirow[t]{2}{*}{ Gender**$^{\star *}$} & Male & $507,246(62.4)$ & $40,536(61.8)$ & $106,164(62.9)$ & $36,431(61.7)$ & $401,082(62.3)$ & $4,105(63.3)$ \\
\hline & Female & $305,362(37.6)$ & $25,018(38.2)$ & $62,720(37.1)$ & $22,634(38.3)$ & $242,642(37.7)$ & $2,384(36.7)$ \\
\hline \multirow[t]{5}{*}{ Race } & Non-Hispanic White & $199,776(24.6)$ & $13,993(21.3)$ & $49,711(29.4)$ & $12,890(21.8)$ & $150,065(23.3)$ & $1,103(17)$ \\
\hline & Non-Hispanic Black & $359,455(44.2)$ & $34,087(52)$ & $71,323(42.2)$ & $30,347(51.4)$ & $288,132(44.8)$ & $3,740(57.6)$ \\
\hline & Hispanic & $183,684(22.6)$ & $11,470(17.5)$ & $31,667(18.8)$ & $10,306(17.4)$ & $152,017(23.6)$ & $1,164(17.9)$ \\
\hline & Asian & $13,390(1.6)$ & $1,479(2.3)$ & $3,591(2.1)$ & $1,358(2.3)$ & $9,799(1.5)$ & $121(1.9)$ \\
\hline & Other & $56,325(6.9)$ & $4,529(6.9)$ & $12,597(7.5)$ & $4,168(7.1)$ & $43,728(6.8)$ & $361(5.6)$ \\
\hline \multirow[t]{3}{*}{ Payer } & Government & $554,076(68.2)$ & $43,590(66.5)$ & $110,585(65.5)$ & $39,216(66.4)$ & $443,491(68.9)$ & $4,374(67.4)$ \\
\hline & Private & $229,309(28.2)$ & $20,174(30.8)$ & $52,269(30.9)$ & $18,183(30.8)$ & $177,040(27.5)$ & $1,991(30.7)$ \\
\hline & Other & $29,245(3.6)$ & $1,794(2.7)$ & $6,035(3.6)$ & $1,670(2.8)$ & $23,210(3.6)$ & $124(1.9)$ \\
\hline \multicolumn{2}{|c|}{$\begin{array}{l}\text { Intensive Care Unit } \\
\text { Utilization }\end{array}$} & $9,069(1.1)$ & $16,555(25.3)$ & $9,062(5.4)$ & $16,550(28)$ & & \\
\hline \multicolumn{2}{|c|}{ Length of Stay (days) } & $1[1,1]$ & $2[1,2]$ & $1[1,2]$ & $2[1,3]$ & & \\
\hline \multicolumn{2}{|c|}{$\begin{array}{l}\text { Emergency Department Encounter in Prior Year for } \\
\text { Asthma (Admitted or Not) }\end{array}$} & $260,262(32)$ & $23,946(36.5)$ & $53,392(31.6)$ & $21,390(36.2)$ & $206,870(32.1)$ & $2,556(39.4)$ \\
\hline \multicolumn{8}{|c|}{ All comparisons significant at $P<.001$,except where noted. } \\
\hline \multicolumn{8}{|c|}{ * Mag: Intravenous Magnesium } \\
\hline \multicolumn{8}{|c|}{ 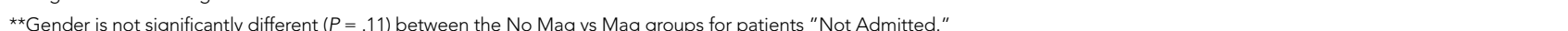 } \\
\hline
\end{tabular}

The PHIS database is an administrative database that contains demographics, International Classification of Diseases 9th and 10th Revision diagnoses and procedures, and daily billing records for all inpatient, observation, ED, and ambulatory surgery encounters. All data were deidentified prior to inclusion in the database and tracking of patients across ED and inpatient visits was achieved through an encrypted and unique patient identifier. Children transferred from other hospitals were excluded because we could not verify IV magnesium use prior to transfer. For hospitals to be included, they were required to provide continuous data throughout the study period.

\section{Main Outcome Measure}

The main outcome was exposure to IV magnesium as determined by billing information available in the PHIS database.

\section{Patient Demographics}

We assessed patients' demographic characteristics, including age (younger than 5 years, 5-11 years, and 12-17 years), sex, race/ethnicity, and insurance status.

Healthcare Utilization and Hospital Characteristics We assessed healthcare utilization using geometric mean LOS, proportion of patients admitted to the hospital and to the ICU, and the proportion of patients with a 7-day all-cause readmission. In addition, we divided hospitals into three equal groups based on their annual inpatient asthma volume $(<300,300-850$, $>850$ cases per year).

\section{Statistical Analysis}

We compared demographic and clinical characteristics across patients receiving IV magnesium with those who did not receive it with use of chi-square tests for categorical variables and Wilcoxon rank sum test for continuous variables. We calculated annual IV magnesium use rates for each hospital and modeled the average annual rate with a general linear model in order to assess change over time. We used Pearson product moment correlation to compare the annual proportion of magnesium use and healthcare utilization measures, including geometric mean LOS, the proportion of patients using the inpatient wards or the ICU, and the proportion of cases with a 7-day all-cause readmission. Geometric mean LOS was used to normalize the compounding effect of non-normally distributed arithmetic mean LOS. A sensitivity analysis was performed stratifying IV magnesium use over time by hospital inpatient volume. Data were analyzed using SAS version 9.4 (SAS Insti- 

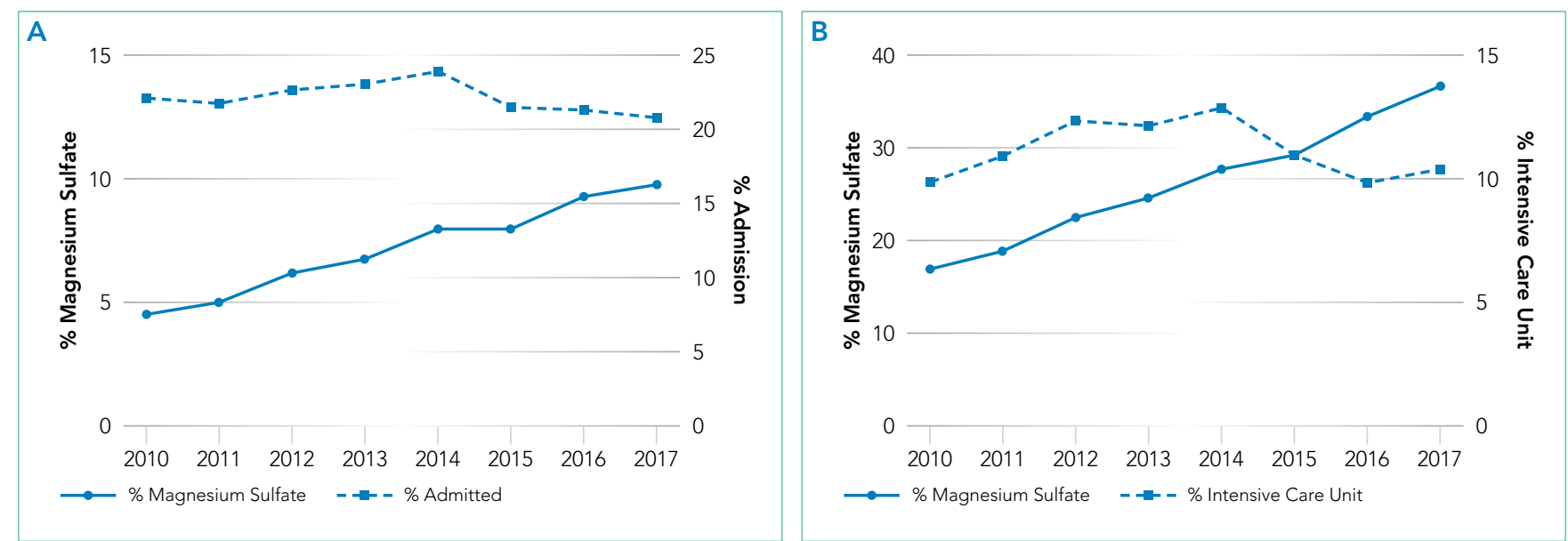

FIG. Trends in Intravenous Magnesium Sulfate Use and Hospital Outcomes at 35 Children's Hospitals, 2010 to 2017. (A) Percentage of patients who received magnesium sulfate and were admitted to hospital. There was an increase in the percentage receiving magnesium sulfate $(P<.001)$ but no change in the percentage admitted $(P=$.34). There was no correlation between the percentage receiving magnesium sulfate and the percentage admitted to hospital over the study period $(P=.46)$. (B) Percentage of patients who received intravenous magnesium sulfate and were hospitalized in the Intensive Care Unit (ICU). There was an increase in the percentage receiving intravenous magnesium sulfate $(P<.001)$ but no change in the percentage admitted to the ICU $(P=.75)$. There was no correlation between the percentage receiving magnesium sulfate and the percentage admitted to the ICU during the study period $(P=.74)$.

tute, Cary, North Carolina), and $P$ values $<.05$ were considered statistically significant.

\section{RESULTS}

\section{Study Population}

A total of 878,188 encounters with acute asthma exacerbation met the inclusion criteria, with 65,558 (7.5\%) receiving IV magnesium (Table). Of those receiving IV magnesium, $90 \%$ were admitted to the hospital. There were statistically significant differences in IV magnesium use when compared by age, race/ethnicity, and payer type, but not gender. IV magnesium use was significantly associated with older age (more than 5 years old), non-Hispanic black race, ED visit in the year prior to admission, longer hospital LOS, and higher ICU admission rate.

\section{Trends in Intravenous Magnesium Use}

IV magnesium use among hospitalized children more than doubled from 2010 to 2017 (17\% vs 36\%). Low-volume hospitals had a lower frequency of IV magnesium use, compared with the moderate- and high-volume hospitals. The growth rate per year of IV magnesium use was greater in high- and moderate-volume hospitals (3.4\% and $2.9 \%$ per year, respectively), compared with the low-volume hospitals (1.2\% per year; $P=.04$ ).

\section{Trends in Intravenous Magnesium Use and Hospital Outcomes}

The trend in IV magnesium use was not associated with a statistically significant change in the inpatient and ICU admission rate or in the 7-day all-cause readmission rate (Figure and Appendix Figure). Although IV magnesium use increased over time, LOS decreased significantly during the same period ( 1.6 days in 2010 vs 1.4 days in 2017; $P<.001$ ). When analyzed by hospital volume, no significant associations were found in the inpatient admission, ICU admission, and 7-day readmission rate.

\section{DISCUSSION}

The use of IV magnesium has significantly increased in US children's hospitals over the last 8 years, especially among those hospitalized following an ED evaluation. Over this interval, trends in inpatient and ICU admission rate, as well as 7-day allcause readmission rate, for asthma did not change, while LOS decreased. These findings contrast with a recent Cochrane review that summarized the efficacy of IV magnesium for reducing admission rate in few small trials. ${ }^{2}$

Our study findings are more consistent with prior survey findings that IV magnesium does not reduce hospitalization and that ED physicians tend to use IV magnesium in severe asthma exacerbation for its potential therapeutic benefits because of bronchodilator and anti-inflammatory effect. ${ }^{6,7}$ Similar to PEM physicians' estimates, only $10 \%$ of patients receiving IV magnesium were discharged home in our study.

IV magnesium use is higher in high-volume hospitals than in moderate- and low-volume ones. One potential explanation is that high- and moderate-volume hospitals may see a higher volume of children with severe or impending respiratory failure and, therefore, are more likely to use IV magnesium than the low-volume hospitals are. Alternatively, physician adoption of magnesium use for lower-acuity asthma exacerbations could vary by hospital volume.

Trend analyses of outcomes suggest that increase in IV magnesium use was not associated with an increase in inpatient and ICU admission rate or with 7-day all-cause readmission rate, although LOS reduced. LOS might be reduced because of various quality improvement initiatives (eg, discharging patients after every 3 hours albuterol treatments or respiratory therapist-driven protocols) and might not be related to IV magnesium use.8,9 To this point, a recent study of a respiratory assessment score-matched cohort did not find any therapeutic benefit of IV magnesium with severe asthma exacerbation when receiving continuous albuterol therapy 
on a pediatric ward..$^{5}$ Perhaps future studies could explore estimating the outcome by performing comparative effectiveness studies between those with severe asthma exacerbation who did or did not receive IV magnesium. Additionally, randomized controlled trials comparing IV magnesium and standard therapy and its effects on outcomes, such as hospitalization, LOS, association with asthma chronicity, and previous oral steroid use, might provide further insight to inform clinical practice.

Certain study limitations should be noted. The study cohort included children's hospitals only, and it is possible that care at nonchildren's hospitals for asthma differs. PHIS dataset used in this study does not allow determination of where and when IV magnesium was given, the severity of asthma exacerbation, or the chronicity of baseline disease. Moreover, PHIS hospitals include centers in large cities, and other competing children's hospitals may provide other tertiary care that could affect the readmission data calculation. Lastly, the temporal associations

\section{References}

1. National Asthma Education and Prevention Program, Third Expert Panel on the Diagnosis and Management of Asthma. Expert Panel Report 3: Guidelines for the Diagnosis and Management of Asthma. Bethesda, Maryland: National Heart, Lung, and Blood Institute; 2007. https://www.ncbi.nml.gov/ books/NBK7232/

2. Griffiths $B$, Kew KM. Intravenous magnesium sulfate for treating children with acute asthma in the emergency department. Cochrane Database Syst Rev. 2016;4(4):CD011050. https://doi.org/10.1002/14651858.CD011050.pub2

3. Shan Z, Rong Y, Yang W, et al. Intravenous and nebulized magnesium sulfate for treating acute asthma in adults and children: a systematic review and meta-analysis. Respir Med. 2013;107(3):321-330. https://doi.org/10.1016/j. med.2012.12.001

4. Rower J, Liu X, Yu T, Mundorff M, Sherwin C, Johnson M. Clinical pharmacokinetics of magnesium sulfate in treatment of children with severe acute asthma. Eur J Clin Pharmacol. 2017;73(3):325-331. https://doi.org/10.1007/ s00228-016-2165-3 between IV magnesium use and outcomes reported in this study should not be used as evidence or lack of evidence for the effectiveness of magnesium given the limitations of the observational study design and dataset used.

In conclusion, IV magnesium use in management of asthma exacerbation in children across the United States has significantly increased. The increase occurred disproportionately in high-volume hospitals and was not associated with changes in admission rate, ICU admission rate, or 7-day all-cause readmission rate, although LOS has decreased over time.

Disclosures: The authors have no financial relationships relevant to this article or conflicts of interest to disclose.

This paper was a platform presentation at annual meetings of Pediatric Academic Societies 2019; accepted for presentation at annual meeting of Pediatric Hospital Medicine, July 2019.

Funding Source: No funding was secured for this study.

5. Desanti R, Agasthya N, Hunter K, Hussain M. The effectiveness of magnesium sulfate for status asthmaticus outside intensive care unit. Pediatric Pulmonol. 2018;53(7):866-871. https://doi.org/10.1002/ppul.24013.Epub 2018

6. Schuh S, Macias C, Freedman S, et al. North American practice patterns of intravenous magnesium sulfate in severe acute asthma exacerbations. Acad Emerg Med. 2010;17(11):1189-1196. https://doi.org/10.1111/j.1553-2712.2010.00913.x

7. Cheuk DK, Chau TC, Lee SL. A meta-analysis on intravenous magnesium sulphate for treating acute asthma. Arch Dis Child. 2005;90(1):74-77. https:// doi.org/10.1136/adc.2004.050005

8. Lo HY, Messer A, Loveless J, et al. Discharging asthma patients on 3-hour $\beta$-agonist treatments: a quality improvement project. Hosp Pediatr. 2018;8(12):733-739. https://doi.org/10.1542/hpeds.2018-0072

9. Magruder TG, Narayanan S, Walley S, et al. Improving inpatient asthma management: the implementation and evaluation of pediatric asthma clinical pathway. Pediatr Qual Saf. 2017;2(5);e041. https://doi.org/10.1097/ pq9.0000000000000041 